\title{
Research on Quality Measurement and Evaluation Method of the Localization Embedded Real-Time Operating System
}

\author{
GAO ZhiJie ${ }^{a,{ }^{*}}$, ZHANG Youchun, LIU Chen and JIAO Wen \\ No.109 Bei Qing Road Haidian District, Beijing, China \\ azjiegao@163.com
}

Keywords: Localization; embedded; real-time; Operating system.

Abstract. Embedded real-time operating system is the basic support environment for all kinds of embedded systems, which directly determines the quality of the embedded application function, the performance index of the range. To the quality of embedded real-time operating system quantitative measurement and evaluation, based on the current is generally recognized by software quality metrics for the establishment of quality evaluation index system of embedded real-time operating system. The system has six aspects: function, efficiency, reliability, ease of use, maintainability and portability.

\section{Introduction}

Embedded Real-time Operation System (ERTOS) is a special kind of operating system. Real-time operating system is mainly used in the field of industrial control, military equipment, aerospace and other response time of the system has a demanding requirements. System generated in the external events or data, can accept and treat it with fast enough speed, the processing result and within the stipulated time to control the production process or make rapid response to processing system and control all real-time tasks coordinated operation.

At present, the application of embedded real-time operating system in the related fields of our country is mostly foreign products, which is representative of the America Wind River Corporation's VxWorks. But with the continuous advance of China's localization strategy, in recent years, domestic also has launched a domestic embedded real-time operating system products, representative is seven Shenzhou OS developed by China Electronics Technology Group Corporation No. 32 Research Institute developed Ruihua embedded real-time operating system reworks, Beijing Shenzhou Aerospace Software Technology Co., Ltd. and China Aerospace Science and industry group, the Research Institute, six developed star rocket carrying high performance real-time operating system.

With the embedded real-time operating system application in various fields especially the application and extension of military equipment in the field and in the field of industrial control and need of software, especially like embedded real-time operating system so that the basic software for comprehensive assessment and evaluation. Therefore, it is necessary on a set of effective evaluation system, embedded real-time operating system functionality, performance, reliability, portability and other multiple quality factors of comprehensive evaluation, for the development, use and independent evaluation mechanism provides a determined embedded real-time operating system of quality demand and balancing the ability of software on the basis of evaluation.

\section{Features of the Equipment Software}

The equipment software system is an embedded system with high levels of safety critical and mission critical level, real-time operating system, as the system application software provides basic platform, with high safety and reliability. Most weapon equipment software is a typical embedded software, which has the characteristics of embedded software:

1) real-time;

2) physical device correlation; 
3) high reliability;

4) high security.

Among them, real-time requirements of the software to complete the tasks in the course of the war: real-time communications, real-time processing, real-time display, real-time control and real-time decision-making, etc.. According to the different requirements of the real-time requirements of the task can be divided into hard real-time, soft real-time. The so-called hard is that if the time limit is broken once there could lead to disastrous consequences to the system; and soft real-time refers to the time limit by accident by the breakthrough is not sufficient to make the system performance becomes difficult to accept the task.

Physical equipment correlation is refers to the software for embedded computer system as one of its main purpose is not part of a larger computational system can't be separated from the, are associated directly or indirectly with the physical device.

High reliability and high security requirements of this kind of software is absolutely safe and reliable. Under normal circumstances, especially in the case of software or system equipment failure, or software running environment changes, the software must provide reliable security services.

Therefore, the embedded real-time operating system as the basic platform, the quality of the quality of the weapon is more important, it is important to measure and evaluate its quality.

\section{Evaluation Parameters System of Embedded Real-time Operating System}

According to the characteristics of weapon equipment software, and combined with the characteristics of software third party software evaluation, the GJB 5236-2004 military software quality metric, establishment of embedded real-time operating system of quality measurement and evaluation system based on, the localization of real-time operating system evaluation to provide technical support.

\section{Metric Element Selection}

According to the characteristics of embedded real-time operating system, the measurement element of the GJB 5236-2004 military software quality measure is reduced. Metric element selected according to the different quality characteristics evaluation techniques, according to the GJB 2434A-2004 protection software product evaluation, selection, selected metrics and the corresponding evaluation technology mainly includes:

\section{A) Function}

The main evaluation techniques used in the functional metric elements include:

1) function test;

2) unit test.

B) Efficiency

The main evaluation techniques used in the measurement of efficiency are included:

1) execution time performance test;

2) memory performance test;

3) strength test.

C) Portability

The main evaluation techniques used in the portable measurement elements include:

1) programming rule checking;

2) compatibility testing.

\section{Evaluation Index System}

According to the evaluation index system of embedded real-time operating system to the GJB 5236-2004 of military software quality metrics, the definition of internal quality and external quality model as the foundation, combined with real-time operating system with its own characteristics, the definition of embedded real-time operating system evaluation index system, including: functionality, efficiency, reliability, ease of use, maintainability, portability, as follows:

1) functional indicators include: the integrity of the functional implementation; 
2) the efficiency index includes: task switching time, scheduling delay, key interface, interrupt response time, memory allocation of time, priority scheduling delay, signal delay, mutual exclusion semaphores delay;

3) reliability index terms include: fault density, average failure time (MTBF), to resist the wrong operation, the average repair time;

4) usability metrics include: the integrity of the user documentation, providing the integrated development environment for visual;

5) the maintenance of the indicators include: easy analysis;

6) The measure of portability of $F$ includes: adaptability.

\section{Functional Integrity Measurement}

A) measurement method

The evaluation of the missing function is detected and compared with the feature set. Feature set including task management, interrupt / exception management, the clock / timer management, memory management, tracking function, equipment management, document management, network protocol stack, shell and tools, graphics system, partition management, process management of partitions, partition communication management, communication management, health management etc..

B) evaluation index calculation method

Calculation formula: $\mathrm{X}=1-\mathrm{A} / \mathrm{B}$

Among them, $\mathrm{A}$ is the function of the function set in the evaluation, which is detected by $\mathrm{B}$. The range of $\mathrm{X}$ value is: $0<X<1$, the $X$ value is higher.

\section{Efficiency Characteristic Measurement}

As an embedded real-time operating system, its time characteristic is an important index to evaluate the system. It plays a decisive role in the application of the operating system and the environment.

The main two time performance measure of the embedded real-time operating system is as follows:

1) Task Switching Time

A) measurement method

After the event triggered switch, from the current task to stop running, save running state (CPU register content), to load the next task to be running state, the time to start running.

B) evaluation index calculation method

The maximum time, the average time, the shortest time and the distribution of the measurement task switching are measured by the evaluation procedure.

C) evaluation criterion is the absolute measure, the smaller the better.

2) Interrupt Response Time

A) measurement method

Interrupt occurs when the user 's interrupt service routine is executed to handle the interrupt.

B) evaluation index calculation method

The maximum time, the average time, the shortest time and the distribution of the interrupt response is measured by the evaluation program.

C) the evaluation criterion is the absolute value, the smaller the better.

\section{Portability Measurement}

The portability measure is mainly aimed at the behavior of the operator or the system during the system transplantation.

A) measurement method

System faces the complexity of different hardware platforms, that is, the adaptability of the system to the operating environment.

B) evaluation index calculation method

The number of technical points of the system to realize the adaptability of different hardware platforms X.

C) evaluation criteria

The larger the $\mathrm{X}$ value, the more adaptive ability of the system. 


\section{system quality comprehensive evaluation}

Based on the selected measurement element of the metric, the evaluation index system of the metric, combined with the quality characteristics of the measurement results, the system total measurement, it is concluded that a comprehensive evaluation of the quality system. In the comprehensive evaluation, the weight of the metric index should be set up. Weights indicate the specific gravity of the indexes in the characteristics.

Set a feature weighted vector:

$\mathrm{W}=\left(\mathrm{W}_{1}, \mathrm{~W}_{2}, \ldots, \mathrm{W}_{6}\right)$

Among them, $W_{j}$ is the first $j$ to measure the weights, sigma $W_{j}=1$.

Control application needs to map the software to the quality of the software quality characteristics, the metric will be derived from the combination of weight calculation of the score of various characteristics.

In the quality evaluation of the Shenzhou OS, considering the system has the characteristics of fault tolerant parallel, the weight of six quality characteristics is set to: function (0.2) and efficiency (0.2), reliability (0.3), ease of use (0.1) and maintenance (0.1), portability (0.1).

And of star projectile load high performance real-time operating system was used to evaluate the quality, considering the system has the characteristics of high performance, the weight of six quality characteristics is set to: function (0.2) and efficiency (0.3), reliability (0.2) and ease of use (0.1) and maintenance (0.1), portability $(0.1)$.

Different quality characteristics of the score does not necessarily indicate that the quality of the software, because the same measure as the application of the different needs of different results will be different.

\section{Summary}

In nuclear high based of major national science and technology projects "embedded real-time operating system and supporting environment for product evaluation" project, on the localization of the operating system quality metric system lacks the corresponding standards, according to the GJB 2434A-2004 protection software product evaluation, through to the troops GJB 5236-2004 software quality metric "to cut the selected metrics and quality evaluation index system is established, the localization of embedded real-time operating system for comprehensive evaluation. The index system from the functionality, efficiency, reliability, ease of use, maintainability and transplantation of six aspects, for embedded real-time operating system evaluation, quality indexes is comprehensive, has general, applicable to a wide range of characteristics. In the evaluation process of the system's validity and correctness of real-time verification, adjust the weight distribution, forming a product supporting the quality evaluation criteria.

\section{References}

[1] GJB 5236-2004:Military software quality metrics.

[2] GJB 2434A-2004:Military software product evaluation.

[3] ANSI/IEEE 1003.13-2003 POSIX:Real-time embedded application support.

[4] LiQingcheng, Tang Dekai. Real-time comparison and evaluation of embedded real-time operating system [J]. Microcontroller and embedded systems applications, 2012, (6): 18-21.

[5] Zhou Hangyu. Study on the evaluation index system of the embedded real-time operating system[J]. Microcomputer application, 2013 (12): 7-9. 